\title{
PREVALÊNCIA E FISIOPATOLOGIA DA LITÍASE BILIAR EM PACIENTES SUBMETIDOS A TRANSPLANTE DE ÓRGÃOS
}

\author{
Prevalence and physiopathology of gallstone in transplant patients
}

\author{
Júlio Cesar Uili COELHO ${ }^{1}$, Fabiana Loss de CONTIERI ${ }^{2}$, Jorge Eduardo Fouto MATIAS ${ }^{1}$, \\ Mônica Beatriz PAROLIN ${ }^{1}$, Jose Luiz de GODOY ${ }^{1}$
}

\begin{abstract}
Coelho JCU, Contieri FL, Matias JEF, Parolin MB, Godoy JL. Prevalência e fisiopatologia da litíase biliar em pacientes submetidos a transplante de órgãos. ABCD Arq Bras Cir Dig 2009;22(2):120-3

RESUMO - Introdução - O objetivo do presente estudo é apresentar revisão da prevalência e dos principais mecanismos fisiopatológicos que levam a formação da litíase biliar em pacientes submetidos a transplante de órgãos. Métodos - Revisão da literatura abrangendo 29 publicações obtidas das bases Medline/Pubmed, Scielo e Lilacs com cruzamento dos unitermos "transplante, transplante renal, transplante hepático, cálculo biliar, colecistite.". Vários estudos que utilizaram a ultrassonografia demonstraram aumento na prevalência da litíase biliar em pacientes submetidos a transplante de órgãos. A taxa de formação de cálculos novos após o transplante variou de 10 a $55 \%$ e a total (cálculos formados antes e após o transplante) de 17 a $68 \%$. Tanto o ganho como a perda de peso rápida, que podem ocorrer após o transplante, predispõem à formação de cálculos biliares. O uso do imunossupressor ciclosporina é considerado como o principal fator que aumenta a incidência de litíase biliar após o transplante. Conclusão - A prevalência de litíase biliar é maior nos pacientes submetidos a transplante de órgãos do que na população geral. As principais alterações que ocorrem no paciente transplantado que predispõem a formação dos cálculos são as alterações do peso corporal, uso de imunossupressores, diabete melito e hiperlipidemia. DESCRITORES - Transplante. Transplante renal. Transplante hepático. Cálculo biliar. Colecistite.
\end{abstract}

\section{INTRODUÇÃO}

A formação da litíase da vesícula biliar resulta da interação de fatores genéticos e ambientais ${ }^{5}$. A sua prevalência depende de vários fatores e é variável conforme a população. Vários estudos sugerem que ela é maior em pacientes submetidos a transplante de órgãos e tecidos ${ }^{1,11,12,13}$.

O objetivo no presente estudo é apresentar revisão da prevalência e dos principais mecanismos fisiopatológicos que levam à formação da litíase biliar em pacientes submetidos a transplante de órgãos.

\section{MÉTODOS}

Revisão da literatura abrangendo 29 publicações obtidas das bases Medline/Pubmed, Scielo e Lilacs com cruzamento dos unitermos "transplante, transplante renal, transplante hepático, cálculo biliar, colecistite.”.

Trabalho realizado no Serviço de Transplante Hepático do Hospital de Clínicas da Universidade Federal do Paraná ${ }^{1}$ e Serviço de Transplante Renal do Hospital Universitário Evangélico de Curitiba/Faculdade Evangélica do Paraná2, Curitiba, PR, Brasil.

Endereço para correspondência: Júlio Coelho, e-mail: coelhojcu@yahoo.com.br

\section{Prevalência da litíase biliar}

População geral

A litíase biliar é uma das condições mais comuns e sua prevalência varia com o país e certos grupos populacionais estudados. Na maioria dos países Europeus, Estados Unidos e Canadá, ela é de cerca de $10 \%$ da população ${ }^{5,8,9}$. É menor na Ásia e África e maior no Chile ${ }^{5}$. No Brasil, os autores deste trabalho já tinham determinado, através de avaliação ultrassonográfica da população de Curitiba, que a prevalência de colelitíase é de 9,3\% em indivíduos com mais de 20 anos de idade ${ }^{6}$. Considerando que a população brasileira é de cerca de 190 milhões de habitantes e que, desses, 105 milhões têm idade igual ou acima de 20 anos, estima-se que 10 milhões de brasileiros com mais de 20 anos de idade apresentam litíase biliar.

Exemplos de variações extremas na prevalência em certos grupos populacionais são os índios pima do estado do Arizona, nos Estados Unidos e os índios masai do leste da África. Com a idade, a maioria dos índios pima apresenta litíase biliar e cerca de $70 \%$ dessas mulheres com idade acima de 25 anos também ${ }^{8}$. Ao contrário, a prevalência de litíase biliar nos índios masai é próxima de zero ${ }^{2}$. 


\section{Pacientes submetidos a transplante de órgãos}

Apesar da prevalência de litíase biliar em candidatos a transplante de órgãos (TO) ser similar ao da população geral, ela aumenta substancialmente após o transplante ${ }^{14,16,19,20,23}$. Entretanto, devido à falta de estudos com grande série de pacientes com controle, que foram submetidos à ultrassonografia no pré e pós-transplante, a verdadeira incidência de cálculos formados após o transplante é difícil de determinar ${ }^{16}$. Além do mais, os poucos estudos disponíveis não comparam a formação de novos cálculos no período pós-transplante com relação à população geral com idade, sexo e índice de massa corpórea similares. Outro fator limitante dos estudos disponíveis é o período de seguimento curto dos pacientes após o transplante ${ }^{23,25}$.

A Tabela 1 mostra os resultados dos estudos que realizaram a ultrassonografia em todos ou quase todos pacientes para determinar a prevalência de litíase biliar em pacientes submetidos a TO. Estes estudos sugerem que existe aumento na prevalência de litíase biliar em pacientes submetidos a ele. Nesta tabela é evidenciada tanto a prevalência de novos cálculos (cálculos formados após o TO) como a prevalência total (cálculos formados antes e após o TO). A taxa de formação de cálculos novos após TO variou de 10 a $55 \%$ e a total de cálculos de 17 a $68 \% \%^{18,23,24,26,27,28}$. Esta ampla diferença se deve, provavelmente, ao período de seguimento variável dos estudos e ao pequeno número de pacientes avaliados. Estudos dos pacientes submetidos a transplante hepático não são disponíveis devido à realização de rotina de colecistectomia durante a retirada do fígado do doador.

TABELA 1 - Prevalência de litíase biliar em pacientes submetidos a transplante de órgãos

\begin{tabular}{lllll}
\hline Autor & Órgão & $\mathrm{N}$ & $\begin{array}{l}\text { Prevalência de cál- } \\
\text { culos novos (\%) }\end{array}$ & $\begin{array}{l}\text { Prevalência } \\
\text { Total (\%) }\end{array}$ \\
\hline Lowell $^{18}$ & Rim/pâncreas & 72 & 30 & 36 \\
Peterseim $^{23}$ & Coração & 60 & 18 & 45 \\
Richardson $^{24}$ & Coração & 518 & 55 & 68 \\
Sarkio $^{26}$ & Pâncreas & 1608 & 10 & 17 \\
Schiemann $^{27}$ & Rim/pâncreas & 83 & 10 & 38 \\
Spes $^{28}$ & Coração & 29 & 33 & 38 \\
\hline
\end{tabular}

\section{Fisiopatologia da litíase biliar}

\section{População geral}

Os principais fatores que participam na formação da litíase biliar são a alteração na composição da bile, a redução na motilidade da vesícula biliar (estase biliar) e a presença de muco e de cálcio na vesícula ${ }^{5}$. A formação de cristais devido à bile litogênica e o aprisionamento deles no muco vesicular, associado à estase na vesícula biliar (hipomoitilidade), levam à formação de cálculos ${ }^{5}$.

A alteração primária que causa a formação de todos os tipos de cálculos biliares é a secreção de bile litogênica pelo fígado ${ }^{5}$. A supersaturada ou litogênica caracteriza-se pelo aumento de colesterol (cálculos de colesterol) ou de bilirrubinato de cálcio (cálculos pigmentares) acima da sua solubilidade.

\section{Cálculo de colesterol}

Colesterol, fosfolipídeos (lecitina) e bilirrubina não conjugada são insolúveis em água. Para serem solúveis é necessário a formação de micelas, que são agregados polimoleculares de sais biliares, fosfolipídios e colestero ${ }^{15}$. Além de solúveis em água, os sais biliares são detergentes, ou seja, são capazes de solubilizar moléculas insolúveis em água, como o colesterol, lecitina e bilirrubina não conjugada.

A solubilidade de colesterol na bile depende da concentração relativa de colesterol, sais biliares e fosfolipídio (lecitina). Quando a sua quantidade aumenta ou as de sais biliares ou lecitina diminuem, a bile torna-se hiper-saturada em colesterol e é denominada bile litogênica ${ }^{5}$.

A secreção dessa bile pelo fígado não é a única condição necessária para formar cálculos, já que mais de $50 \%$ dos indivíduos normais secretam bile super-saturada em colesterol (bile litogênica) após uma noite de jejum ${ }^{5}$. Outras alterações, como estase e secreção de muco, parecem ser importantes na formação dos cálculos biliares ${ }^{5,22}$. Excesso de muco na vesícula biliar pode servir como núcleo para formação de cálculo biliar e a estase na vesícula biliar pode levar à cristalização. Os cristais são fixados e aglutinados na mucina. $\mathrm{O}$ cálcio parece também desempenhar um papel importante na formação dos cálculos, já que sais inorgânicos e orgânicos de cálcio são encontrados nos dois tipos de cálculos ${ }^{5}$. $\mathrm{O}$ cálcio parece reduzir a solubilidade de colesterol e bilirrubina não conjugada. Os sais de cálcio podem também iniciar a formação de um cálculo, agindo como um núcleo para precipitação de outros componentes da bile.

\section{Cálculo pigmentar}

A bilirrubina excretada na bile é quase totalmente do tipo conjugada (solúvel). Na presença de hemólise, a quantidade dela pode aumentar até 10 vezes. A não conjugada (insolúvel) representa apenas $1-2 \%$ da quantidade total de bilirrubina da bile hepática ou da vesícula biliar normal ${ }^{5}$. A maior parte desta bilirrubina é solubilizada em micelas.

Nos pacientes com cálculos pigmentares existe aumento na quantidade de bilirrubina não conjugada na bile devido à transformação de bilirrubina direta (solúvel) em bilirrubina indireta (insolúvel) pela ação de beta-glucuronidase ${ }^{5}$.

\section{Pacientes submetidos a transplante de órgãos}

Além dos mecanismos observados na população geral, os pacientes submetidos a $\mathrm{TO}$ apresentam alterações relacionadas ao transplante, que predispõem à formação de cálculos biliares. As principais alterações relacionadas ao transplante são modificações do peso corporal, diabete melito, imunossupressores e hiperlipidemia ${ }^{3,16,25}$.

Alterações significativas do peso corporal são comuns após TO. Ganho de peso é frequentemente associado ao uso de corticóide e a melhora do apetite devido à correção da insuficiência grave do órgão transplantado. Entretanto, não é incomum a perda de peso secundária à complicações pós-operatórias, clínicas ou cirúrgicas. Complicações clínicas como processos infecciosos e diarréia associadas a imunossupressores geralmente causam perda de peso. Complicações cirúrgicas como infecções e fístulas geralmente são 
associadas a jejum prolongado, aumento do metabolismo e consequentemente perda de peso rápido e intenso ${ }^{11,12}$. Tanto a obesidade como a perda de peso rápida predispõem a formação de cálculos biliares. A incidência de colelitíase aumenta 2 a 4 vezes em pacientes com índice de massa corpórea superior a 40, quando comparado a indivíduos não obesos ${ }^{29}$. A perda de peso rápida, como a observada no pós-operatório de procedimentos cirúrgicos bariátricos, aumenta acentuadamente a formação de cálculos biliares ${ }^{10}$. Miller et al. ${ }^{21}$ relataram o aparecimento de colelitíase em 30\% dos pacientes com obesidade mórbida submetidos ao tratamento cirúrgico em período de seguimento de 24 meses.O principal mecanismo de formação de cálculos biliares em pacientes com ganho de peso ou perda de peso rápida é o aumento da excreção de colesterol na bile, tornando-a supersaturada em colesterol (bile litogênica) ${ }^{5}$. Além disso, essa perda, associada ao jejum prolongado, também predispõe à formação de cálculos biliares por redução da motilidade da vesícula biliar ${ }^{5}$.

A incidência de diabete é maior em pacientes transplantados devido ao uso de imunossupressores. Nos diabéticos a prevalência de litíase é maior devido à redução na motilidade (contratilidade) da vesícula biliar e aumento na excreção de colesterol na bile (bile litogênica) ${ }^{5}$. Lowell et al. ${ }^{18}$ observaram que a incidência de litíase biliar era maior em pacientes submetidos a transplante renal com diabete melito $(27,3 \%)$ do que em não diabéticos $(12,2 \%)$. Na experiência desses autores, a incidência de litíase biliar era também elevada (30,4\%) em pacientes diabéticos submetidos a transplante pancreático.

Alguns trabalhos publicados na década de 80 relataram que o uso de ciclosporina em pacientes submetidos a transplante renal e cardíaco causava aumento de enzimas hepáticas (colestase hepática) e de cálculos biliares e que estas alterações eram dose-dependentes ${ }^{17}$. Muitos autores consideram o uso do imunossupressor ciclosporina como o principal fator que aumenta a incidência de litíase biliar pós-TO ${ }^{13}$. Lorber et al. ${ }^{17}$ relataram a incidência de litíase biliar elevada em pacientes transplantados que receberam ciclosporina. Ao contrário, estes autores observaram que nenhum transplantado que recebeu somente azatioprina e corticóide, desenvolveu litíase biliar. Em estudo em ratos,
Chan e Shaffer ${ }^{4}$ observaram que a ciclosporina possivelmente aumenta a incidência de formação de cálculo biliar de colesterol tanto pela redução do fluxo de bile como pela redução da produção e consequentemente da secreção de sais biliares. A bile torna-se litogênica (aumento relativo do colesterol na bile), devido à redução na excreção de sais biliares, sem alteração na secreção biliar de colesterol. $\mathrm{O}$ desenvolvimento de litíase biliar correlaciona-se tanto com a dosagem, como com a duração da administração de ciclosporina1. Alberú et al. ${ }^{1}$ observaram que a taxa de litíase biliar após transplante renal em pacientes que não receberam ciclosporina foi de $8 \%$. Nos que a receberam por menos 2 anos foi de $16 \%$, e nos por mais de 2 anos de $22 \%$. Ao contrário da ciclosporina, o tacrolimo (FK 506) causa aumento na proporção de ácido quenodeoxicólico e redução na proporção de ácido cólico e assim tem efeito hepatóxico mínimo3. Schiemann et al. ${ }^{27}$ observaram que a prevalência de litíase biliar era menor em pacientes transplantados de pâncreas e rim que receberam tacrolimo do que ciclosporina como agente imunossupressor de primeira linha.

Hiperlipidemia (aumento de colesterol total e triglicerídeos) é um efeito colateral comum de vários imunossupressores, como corticosteróide, ciclosporina, tacrolimo e rapamicina ${ }^{13}$. A azatioprina e o micofenolato mofetil não têm efeito sobre o metabolismo das lipoproteínas. Outras causas de hiperlipidemia em transplantados de órgãos incluem ganho de peso no pós-transplante, diabete melito e uso de medicamentos como de diuréticos e beta-bloqueadores. Alguns autores sugerem que a hipertrigliceridemia e hipercolesterolemia secundárias ao uso de imunosupressores possam desempenhar papel importante na formação de litíase biliar em pacientes transplantados de órgãos ${ }^{13}$.

\section{CONCLUSÕES}

Os dados obtidos da revisão da literatura sugerem que a prevalência de litíase biliar é maior nos pacientes submetidos a TO do que na população geral. As principais alterações que ocorrem e que predispõem a formação de cálculos biliares são as alterações do peso corporal, uso de imunossupressores, diabete melito e hiperlipidemia. 
Coelho JCU, Contieri FL, Matias JEF, Parolin MB, Godoy JL. Prevalence and physiopathology of gallstone in transplant patients. ABCD Arq Bras Cir Dig 2009;22(2):120-3

ABSTRACT - Introduction - The objective of the present study is to review the prevalence and the main physiopathologic mechanisms that result in gallstone formation in transplant patients. Methods - Literature review with 29 papers included in Medline/Pubmed, Scielo and Lilacs database, crossing key-words "transplantation; kidney transplantation; liver transplantation; gallstone; cholecystitis". Several ultrasonographic studies have demonstrated an increase in gallstone prevalence in transplant patients. The formation rate of new stones after transplantation varied from 10 to $55 \%$ and the rate of total stones (stone formed before and after transplantation) varied from 17 to $68 \%$. Both weight gain and rapid weight loss that may occur after transplantation predispose to gallstone formation. Cyclosporine is considered the most important factor in the development of gallstone after transplantation. Conclusion - The prevalence of gallstones is higher in transplant patients. The main risk factors are change in body weight, use of immunosuppressors, diabetes mellitus, and hiperlipidemia.

HEADINGS - Transplantation. Kidney transplantation. Liver transplantation. Gallstone. Cholecystitis.

\section{REFERÊNCIAS}

1. Alberú J, Gatica M,Cachafeiro-Vilar M, Robles-Diaz G, Bezaury P, Paz-Pinedo F,Vargas-Vorachova F. Asymptomatic gallstones and duration of cyclosporine use in kidney transplant recipients. Rev Invest Clin 2001;53:396-400.

2. Biss K, Ho KJ, Mikkelson B. Some unique biologic characteristics of the Masai of East Africa. N Engl J Med 1971;284:694-5.

3. Cao S, Cox K, So SS, Berquist W, Lee SP, Haigh WG, Concepcion W. Potential effect of cyclosporine A in formation of cholesterol gallstones in pediatric liver transplant recipients. Dig Dis Sci 1997;42:1409-15.

4. Chan FK, Schaffer EA. Cholestatic effects of cyclosporine in the rat. Transplantation 1997;63:1574-8.

5. Coelho JCU. Litíase vesicular e colecistite crônica calculosa. In Coelho JCU. Aparelho Digestivo. Clínica e Cirurgia. São Paulo, Atheneu, 2005. P.1662-78.

6. Coelho JCU, Bonilha R, Pitaki SAM, Cordeiro RMV, Salvalaggio PRO, Bonin EA. Prevalence of gallstone in a Brazilian population. Int Surg 1999;84:25-8.

7. Coelho JCU, Matias JEF, Baretta GAP, Celli A, Pisani JC, Yokochi JM. Complicações biliares pós-transplante hepático intervivos. Rev Col Bras Cir 2005;32:195-200

8. Everhart JE, Khare M, Hill M. Prevalence and ethinic differences in gallbladder disease in the United States. Gastroenterology 1999;117:632-8.

9. Ferreira AC, Mauad Filho F, Mauad FM, Barra DA, Mattos RL, Jorge Filho I. Litíase vesicular assintomática em mulheres: aspectos epidemiológicos e clínicos. Rev Col Bras Cir 2006;33:235-41.

10. Freitas ACT, Campos ACL, Parolin MB, Coelho JCU. Análise dos resultados da operação de Fobi-Capella através do sistema Baros. Arq Bras Cir Dig 2006;19:58-62.

11. Freitas ACT, Campos ACL, Malafaia O, Matias JEF, Coelho JCU. Metologia do transplante de ilhotas de Langerhans em camundongos. Arq Bras Cir Dig 2004; $17: 83-6$

12. Greenstein SM, Katz S, Sun S, Glicklich D, Schechner R, Kutcher R, Tellis V. Prevalence of asymptomatic cholelithiasis and risk of acute cholecystitis after kidney transplantation. Transplantation 1997;63:1030-2.

13. Gueguem Y, Ferran L, Souidi M, Batt A-M, Lutton C, Siest G, Visvikis S. Compared effect of immunosuppressive drugs cyclosporine $\mathrm{A}$ and rapamycin on cholesterol homeostasis key enzymes CYP27A1 and HMG-CoA reductase. Basic Clin Pharmacol Toxicol 2007;100:392-7.

14. Jackson T, Treleaven D, Arlen D, D'Sa A, Lambert K, Birch DW. Management of asymptomatic cholelithiasis for patients awaiting renal transplantation. Surg Endosc 2005;19:510-3.

15. Kao LS, Flowers C, Flum DR. Prophylactic cholecystectomy in transplant patients: a decision analysis. J Gastrointest Surg 2005;9:965-72.
16. Kao LS, Kuhr CS, Flum DR. Should cholecystectomy be performed for asymptomatic cholelithiasis in transplant patients? J Am Coll Surg 2003;197:302-12.

17. Lorber MI, Van Buren CT, Flechner SM. Hepatobiliary complications of cyclosporine therapy following renal transplantation. Transplant Proc 1987;19:180810.

18. Lowell JA, Stratta RJ, Taylor RJ. Cholelithiasis in pancreas and kidney transplant recipients with diabetes. Surgery 1993;114:858-63.

19. Menegaux F, Dorent R, Tabbi D, Pavie A, Chigot J-P, Grandjbakhch I. Biliary surgery after heart transplantation. Am J Surg 1998;175:320-1.

20. Milas M, Ricketts R, Amerson JR, Kanter K. Management of biliary tract stones in heart transplant patients. Ann Surg 1996;223:747-56.

21. Miller K, Hell E, Lang B, Lengauer E. Gallstone formation prophylaxis after gastric restrictive procedures for weight loss: a randomized double-blind placebo-controlled trial. Ann Surg 2003;238:697-702.

22. Patankar R, Ozmen M, Bailey I, Johnson C. Gallbladder motility, gallstones, and the surgeon. Dig Dis Sci 1995;40:2323-35.

23. Peterseim DS, Pappas TN, Meyers CH. Management of biliary complications after heart transplantation. J Heart Lung Transplant 1995;14:623-31.

24. Richardson WS, Surowiec WJ, Carter KM, Howell TP, Mehra MR, Bowen JC. Gallstone disease in heart transplant recipients. Ann Surg 2003;237:273-6.

25. Sakopoulos AG, Gundry S, Razzouk AJ, Andrews HG, Bailey LL. Cholelithiasis in infant and pediatric heart transplant patients. Pediatr Transplantation 2002;6:231-4.

26. Sarkio S, Salmela K, Kyllonen L, Rosliakova M, Honkanen E, Halme L. Complications of gallstone disease in kidney transplantation patients. Nephrol Dial Transplant 2007;22:886-90.

27. Schiemann U, Ferhat A, Götzberger M, Kaiser C, Stiel J, Landgraf R, Dieterle C. Prevalence of cholecystolithiasis and its management among kidney/pancreas- transplant type 1 (insulin-dependent) diabetic patients. Eur J Med Res 2008; 13:127-30.

28. Spes CH, Angermann CE, Beyer RW. Increased incidence of cholelithiasis in heart transplant recipients receiving cyclosporine therapy. J Heart Transplant 1990;9:404-7.

29. Uy MC, Talingdan-Te MC, Espinosa WZ, Daez ML, Ong JP. Ursodeoxycholic acid in the prevention of gallstone formation after bariatric surgery: a metaanalysis. Obes Surg 2008;18:1532-8.

Fonte de financiamento: não há Conflito de interesse: não há Recebido para publicação: 05/02/2009 Aceito para publicação: 22/03/2009 